\title{
Quaderni
}

QUADERNI Communication, technologies, pouvoir

89 | Hiver 2015-2016

Penser l'avenir : le CESTA, un think tank atypique

\section{Le CESTA et sa vision prospective : une fonction d'éclaireur toujours indispensable dans le domaine de la santé}

Jean-Pierre Thierry

\section{(2) OpenEdition \\ Journals}

Édition électronique

URL : http://journals.openedition.org/quaderni/956

DOI : $10.4000 /$ quaderni.956

ISSN : 2105-2956

Éditeur

Les éditions de la Maison des sciences de l'Homme

\section{Édition imprimée}

Date de publication : 5 janvier 2016

Pagination : 55-64

Référence électronique

Jean-Pierre Thierry, "Le CESTA et sa vision prospective : une fonction d'éclaireur toujours

indispensable dans le domaine de la santé », Quaderni [En ligne], 89 | Hiver 2015-2016, mis en ligne le

05 janvier 2018, consulté le 20 avril 2019. URL : http://journals.openedition.org/quaderni/956 ; DOI :

10.4000/quaderni.956 


\section{Le CESTA \\ et sa vision prospective : une fonction d'éclaireur toujours indispensable dans le domaine de la santé \\ Jean-Pierre Thierry}

Médecin de Santé Publique

Consultant
Au début des années 1980, le Centre d'Études des Systèmes et des Technologies Avancées (CESTA) a abordé un ensemble de problématiques sur les nouvelles technologies dans un très grand nombre de secteurs d'activités publiques et privées. Parmi eux une place significative était faite au secteur de la santé et en particulier aux problèmes multiples que soulevaient l'introduction et la diffusion de ces technologies à l'hôpital, à la fois en termes d'organisation et d'économie mais aussi dans une perspective industrielle. Dans ce cadre particulier le CESTA a pu jouer un rôle complexe, à la fois lieu d'information et de formation, espace de dialogue et de réflexion mais aussi réservoir d'idées où s'élaborait entre les acteurs une vision possible des développements futurs des technologies hospitalières à l'horizon 2000. Le CESTA en tant qu'organisation ayant une vision et une mission répondait à un besoin qui s'avère tout aussi pressant trente ans plus tard.

L'approche originale mise en œuvre et influencée par les travaux et la pensée originale d'Yves Stourdzé en matière de communication et de système d'information est également toujours aussi féconde. La situation actuelle de l'informatique de santé en France peut en effet bénéficier d'une relecture qui permet de souligner le rôle toujours aussi important du « contrôle centralisé de la communication » dans la genèse des projets. Elle renvoie à la problématique du pouvoir dans la société française, à l'origine de blocages qui semblent perdurer sur longue période. À l'orée d'une transformation assez radicale de la recherche médicale, à commencer par la cancérologie, grâce à l'apport du Big Data qui permettra l'émergence d'une véritable médecine de précision, l'approche systémique et multidisciplinaire 
du CESTA pourrait encore aujourd'hui jouer un rôle d'éclaireur indispensable.

\section{Le CESTA comme « think tank »}

La participation d'experts d'horizons différents permettait de miser sur l'interdisciplinarité grâce à des échanges facilités par la petite taille du centre et misant sur la personnalité des acteurs réunis par Yves Stourdzé. La diversité des parcours et des origines professionnelles était une caractéristique du CESTA.

Ainsi, il était possible d'étudier le développement de l'imagerie médicale dans un cadre permettant de se familiariser avec des secteurs d'applications aussi variées que la robotique, l'enseignement ou encore les jeux vidéo. Une volonté d'ouverture vers l'international permettait de tenir compte des « mouvements » stratégiques, au premier rang ceux des États-Unis. Dans l'activité « Sciences du Vivant » dirigée par Marc Chopplet, la mise en œuvre dans des délais assez courts, d'un cycle de conférence baptisé « Technologies Nouvelles à l'Hôpital » est à considérer comme un « actif » de l'activité de la petite équipe constituée de l'auteur de ses lignes, d'un directeur d'hôpital', d'un chercheur en sciences sociales ${ }^{2}$ et d'une assistance administrative ${ }^{3}$.

Les travaux du CESTA ont pu associer les différents partenaires (ministères de la Recherche et de la Technologie, ministère de la santé, industriels, représentants des professionnels de Santé et notamment de la médecine universitaire). Le positionnement institutionnel de l'organisme permettait de compter sur la présence de cadres d'administrations centrales (Direction des hôpi- taux du ministère de la Santé par exemple). Le CESTA pouvait privilégier une approche décomplexée de la prospective qui mobilisait l'expertise, la culture technique et la capacité d'analyse critique des politiques en matière d'innovation grâce à une approche interdisciplinaire. Une position à la fois critique et dynamique pouvait être ainsi prise sans occulter l'importance des grands programmes internationaux qu'il n'avait pas peur de mettre en perspective (la robotique au Japon, la recherche de suprématie américaine, etc.). Proche du mouvement du «Technology Assessment » inauguré par les États-Unis dans les années 70, les travaux du CESTA ont été d'une durée trop courte pour démontrer tout leur intérêt en matière de politique de santé. D'autres facteurs de blocage auraient cependant sans doute freiné son développement, tout comme aux États-Unis après les décisions de dérégulation initiés dès 1980 et qui ont notamment abouti à la disparition de l'OTA (Office of Technology Assessment) en 1995.

\section{D'une politique volontariste à un renoncement industriel}

Entre 1983 et 1987, cinq ou six journées « Technologies Nouvelles à l'Hôpital » ont été organisées dans l'amphi Poincaré, au rez-de-chaussée de l'aile du bâtiment Foch abritant le CESTA. Les échanges interdisciplinaires et intersectoriels n'étaient pas communs dans des réunions publiques thématiques. TNH facilitait les rencontres avec les dirigeants d'entreprise et les chercheurs du secteur biomédical et de l'informatique de santé. Parmi les thèmes abordés, l'imagerie médicale a pris une place de choix (Imagerie par Résonance Magnétique, Échographie) mais d'autres thèmes ont permis d'aborder des problé- 
matiques originales qui sont toujours d'actualité 30 ans plus tard comme avec les journées TNH sur le risque infectieux en imagerie médicale. Co-organisées avec le service de radiologie de l'Institut Pasteur ${ }^{4}$ dirigé par le Professeur François Gros par ailleurs Président du conseil scientifique du CESTA, ces journées TNH ont permis d'attirer l'attention sur le risque infectieux associé à l'usage des sondes d'échographie. Trente ans plus tard, il s'agit d'un dossier toujours d'actualité en France ${ }^{5}$.

Ce type d'organisation peut paraître banal aujourd'hui quand on assiste à la multiplication des rencontres, symposium et séminaires réunissant les intervenants des différents secteurs. Replacées dans le contexte de l'époque, elles avaient quelque chose de vraiment novateur et s'inscrivaient assez naturellement comme prolongement de l'impulsion donnée par les nouvelles orientations vouées à la recherche médicale à partir de 1981. Il s'agissait notamment, avec le concours du ministère de la Recherche, de tenter de promouvoir l'industrie biomédicale française, ou plutôt d'ailleurs de la redynamiser. Ainsi, les « TNH » sur l'imagerie médicale réunissaient la fine fleur de l'industrie et des chercheurs français intéressés par les transferts de technologie et la valorisation (avec la Thomson CGR, le CEA, Supelec et quelques PME dont EDAP dans le domaine de l'échographie). En 1983, plusieurs grands groupes industriels français avaient déjà renoncé à poursuivre dans le biomédical (Dassault dans le monitorage cardiaque par exemple) et seule la branche médicale de la Thomson faisait encore apparaître un Français aux côtés des « grands de l'électronique » tels que General Electric, Siemens et Philips dont les branches médicales continuent de prospérer aujourd'hui' ${ }^{6}$. Autre détail assez intéressant, le CESTA a assez tôt manifesté son intérêt pour le rôle que pouvait jouer le capital risque dans l'émergence d'une offre industrielle tirant partie de la valorisation de la recherche. Une étude financée par le CESTA a cependant permis de mettre en évidence le faible engagement des fonds communs de placement à risque français pour un secteur souffrant d'une assez mauvaise réputation ${ }^{7}$. Une étude avait également été commandée sur la valorisation de la recherche aux États-Unis. Réalisée par un chercheur français en poste au $\mathrm{NIH}^{8}$, elle permettait d'anticiper sur l'accélération de la valorisation aux États-Unis suite à la promulgation, en 1980, du Bay-Dhole Act.

Le CESTA était donc bien positionné pour jouer un rôle dans l'analyse des enjeux de la filière biomédicale tout en intégrant les aspects liés à l'organisation du système de santé. La question de l'équipement du parc français en modalités modernes d'imagerie médicale (Scanners, IRM) était l'un des thèmes privilégiés. Les travaux de veille de la structure permettaient de mettre en avant les risques de retard avec leurs conséquences. Les relations entre le mode opératoire de « contrôle » de l'équipement et donc le rôle de la planification centrale dans la gestion des autorisations d'équipement étaient analysées. La cause principale du retard de la diffusion des scanners crâniens et corps entiers dans l'attente de la mise au point des équipements français, avait été identifiée. L'étude de l'émergence, dans les années 1980, de la nouvelle modalité phare d'imagerie, l'Imagerie par Résonance Magnétique - IRM - était un objet de recherche assez 
approfondie qui a pu bénéficier du travail de deux élèves polytechniciens encadrés par le CESTA pour écrire une « micro-thèse » sur ce thème ${ }^{9}$. Il était ainsi possible de voir à quel point les orientations technologiques en rapport avec la question de la «puissance » des aimants équipant ces machines empruntaient plus au marketing qu'à la science dure. Une collaboration avec des organisations médicales spécialisées a ainsi permis de commencer à analyser plus en détail le paysage sanitaire français. Cela s'est par exemple traduit par la publication d'un « panorama de la neuroradiologie française » en collaboration avec la Société Française de Neuroradiologie présidée à l'époque par le $\mathrm{Pr}$ Jean-Marie Caillé, chef de Service au CHU de Bordeaux.

D'autres thèmes, depuis devenus " porteurs ", étaient abordés, par exemple lors des journées TNH sur la biologie médicale. Inspiré notamment par les travaux du Pr Sultan (Hôpital Henri Mondor, APHP, Paris), elles ont permis d'explorer le potentiel de l'informatisation des plateaux techniques en mettant déjà en avant l'importance que celle-ci pourrait prendre afin de favoriser une rationalisation de la prescription. On parlait déjà à l'époque au CESTA de pertinence des actes et des prescriptions, un thème qui revient actuellement sur le devant de la scène dans des systèmes de santé confrontés désormais à des pressions économiques croissantes, voire menacés de non-soutenabilité économique.

Moins de trois ans après la fermeture du CESTA, l'annonce de la vente de l'activité d'imagerie médicale de la Thomson à General Electric a entériné d'une certaine façon le renoncement de la France à engager une politique ambi- tieuse dans le secteur biomédical. S'il n'est pas utile de revenir sur les causes profondes du renoncement français, force est de constater que le mouvement de désindustrialisation était déjà bien engagé. Le déficit commercial annuel de ce secteur dépasse aujourd'hui les 2 milliards d'euros et, trente ans plus tard, des mesures de relance sont toujours proposées ${ }^{10}$.

La disparition du CESTA a coïncidé également avec le vaste mouvement de dérégulation qui a abouti dans de nombreux pays à favoriser une absence de contrôle effectif de la diffusion des innovations médicales dans les trois décennies qui ont suivi ; l'innovation étant le véritable moteur de la croissance des dépenses de santé, beaucoup plus que le vieillissement de la population qui a un impact en réalité modeste. Dès 1980 aux États-Unis, les premières attaques sur l'Office of Technology Assessment étaient portées par les Républicains et par leurs officines ${ }^{11}$. La suppression du CESTA, sans représenter une décision de même portée, peut cependant être rapprochée de l'entrée dans la période de dérégulation de l'ère Reagan-Thatcher. L'approche systémique et par trop intellectuelle des technologies conduites par l'OTA aux États-Unis et, plus modestement par le CESTA, s'opposaient sans doute aux nouveaux dogmes.

Trente ans plus tard, le retour de l'évaluation des technologies médicales est préconisé par plusieurs États-membres et par la Commission Européenne. Les rencontres réunissant administratifs, spécialistes médicaux, journalistes, médecins, industriels, se sont multipliées au point de constituer, pour leurs organisateurs, un modèle d'affaires en tant que tel. Aucun, ou presque, ne 
peut garantir un cadre à la fois libre et suffisamment institutionnalisé. Ces réunions semblent avant tout construites pour « influencer » les décideurs qui acceptent d'ailleurs volontiers d'y participer. La période actuelle est caractérisée par la multiplication des sources d'informations et des réunions avant tout financées par des sponsors où la recherche d'une pensée critique et systémique fait largement défaut. Les journées TNH du CESTA n'ont sans doute pas d'équivalent aujourd'hui.

\section{Permanence de la vision d'Yves Stourdzé}

La conduite de l'informatisation du système de santé français depuis plusieurs décennies peut être analysée à partir de la grille de lecture que nous a léguée Yves Stourdzé. En partant du mythe d'origine des systèmes de télécommunication décrit par Yves Stourdzé, il est possible encore aujourd'hui d'appliquer la grille de lecture de ce qu'il appelait « l'effet Chappe » pour comprendre les difficultés rencontrées par l'administration française. Le « grand monologue » de l'État avec la société française revient bien à proposer régulièrement un système centralisé et centralisateur.

Il en a été ainsi pour le « Réseau Santé Social », projet déjà oublié ayant débouché sur un contrat de monopole octroyé à un opérateur téléphonique en 2002. Il est en de même pour le projet de « Dossier Médical Personnel » inauguré en 2000 et qui aura couté plus de 500 millions d'euros pour déboucher sur un constat d'échec en 2014. Le projet est désormais repris par la Caisse Nationale d'Assurance Maladie des Travailleurs Salariés. Auparavant, le projet de diffusion à grande échelle des cartes à mémoire dans le sec- teur de la santé, démarré dans les années 80 , a été un véritable succès. La Carte Vitale est utilisée par presque tous les Français et les professionnels de santé disposent de leurs propres cartes contrôlant les accès et utilisées conjointement avec la Carte Vitale. Ce projet a pu servir de référence aux porteurs des nouveaux projets plus ambitieux qui ont pensé renouveler le tour de force. Mais remplir un dossier médical correspond à une tâche professionnellement chargée, à la fois symboliquement et en pratique. Le contenu du dossier patient reflète à la fois la pensée médicale et engage la relation avec le patient. Le partage de cette information riche et sensible, il ne s'agit plus ici de seulement dématérialiser des factures, cela induit de profonds changements et revient à instaurer une nouvelle transparence qui n'est pas, historiquement, une caractéristique forte du monde de la santé. Pour ces raisons, la « conduite du changement » accompagnant les projets tels que celui du dossier médical partagé entre professionnels et avec les patients est considéré dans le monde comme le principal facteur de succès. Une adaptation qui nécessite de revoir les modes de financement et faire évoluer les organisations en mobilisant puissamment les différents acteurs « sur le terrain » : patients et professionnels de santé. Le projet de DMP français apparaît comme un projet soumis à « l'effet Chappe ". Sa centralisation reproduit le réseau en étoile des réseaux ferrés nationaux. Une seule solution, supportée par un seul « hébergeur » détenteur de l'autorisation régalienne, pourra donc collecter, gérer et permettre la communication des données concernant l'ensemble de la population.

La pensée et l'analyse de Stourdzé tout aussi pertinentes trente ans plus tard permettent 
d'analyser les conséquences du "fantasme pyramidal ». Mais l'État est en réalité encore plus affaibli, le réseau centralisé qui a gagné la partie n'est pas le sien mais celui de tiers. Les GAFA sont devenus les véritables centralisateurs et des armées d'avocats et de conseils échangent constamment avec les régulateurs tout en tirant leur puissance du marketing et de la globalisation des marchés. L'État tout en essayant de contrôler le territoire national, n'a pas pu anticiper sur les effets de la dérégulation des réseaux à l'échelle mondiale et doit gérer le risque de «disruption ». L'informatisation du système de santé est devenue indispensable, il s'agit désormais de «piloter » une pratique médicale devenue plus complexe avec des données alimentant des systèmes d'aide à la décision. Une meilleure communication des informations est également en mesure de contribuer à augmenter la sécurité des patients : les erreurs médicales sont devenues très fréquentes dans un système devenu excessivement fragmenté et disposant de moyens thérapeutiques puissants mais souvent susceptibles de générer des effets secondaires dangereux. L'iatrogénie est, désormais pour l'OMS, au quatrième rang des causes de mortalité évitables dans le monde ${ }^{12}$. Un meilleur usage des technologies médicales et une meilleure information des professionnels de santé comme des patients, devraient permettre d'améliorer la pertinence et l'efficacité des actes, examens et procédures. La surmédicalisation peut même appeler désormais des réponses urgentes, comme avec les antibiotiques dont l'usage irraisonné (en médecine mais aussi dans l'industrie agro-alimentaire) fait désormais courir le risque quasi-apocalyptique d'un retour en arrière à une époque pré-antibiotique.
C'est donc une articulation complexe entre pilotage central, conduite du changement sur le terrain et alignement des modèles de régulation économique qui devrait contribuer à mieux définir toute politique de modernisation des systèmes de santé confrontés aux dangers de la « disruption ». Il s'agit désormais de disposer d'une nouvelle infrastructure informationnelle dont à la fois l'architecture et le mode opératoire ne peuvent être définis qu'au prix d'une démarche éclairée par la prospective et l'analyse systémique. Celle-ci, pour plusieurs raisons, restera avant tout dépendante d'une mise en œuvre par les États, seuls susceptibles d'articuler les dimensions complémentaires (assurance maladie, évolution des pratiques, planification des ressources technologiques, redistribution, protection des usagers). Avec l'émergence de la médecine de précision et donc du besoin de gérer des volumes de données considérables générés notamment par les nouveaux outils de séquençage de l'ADN, des projets nationaux ambitieux voient le jour et ne sont pas sans rappeler la période de la « guerre des étoiles » des années $80^{13}$. Plusieurs pays s'engagent dans le développement d'infrastructures informatiques désormais considérées comme indispensables pour relancer la recherche médicale et notamment la lutte contre le cancer. Le Big Data en médecine et l'introduction de systèmes d'aide à la décision médicale vont nécessiter plus que jamais de pouvoir bénéficier d'une vision prospective et donc de lieux de réflexion et d'animation facilitant l'émergence de nouveaux modèles nécessitant une modification des frontières entre disciplines et, sans doute, la modernisation de certaines citadelles.

Aussi, le dernier mot peut revenir à Yves 
Stourdzé. Alors qu'il se savait atteint par la maladie, Yves Stourdzé continuait à réfléchir au bien commun et nous offrait un éclairage qui garde tout son intérêt aujourd'hui. Dans une note d'avril 1986, Yves Stourdzé mentionne l'importance des travaux multidisciplinaires du CESTA et l'intérêt des journées TNH qui peuvent participer d'une « orthopédie du système de santé, non seulement possible, mais qui constitue une ardente obligation». Yves Stourdzé, au retour d'un voyage qui lui permet de prendre connaissance du programme scientifique de la guerre des étoiles publié par le Général Abrahamson, établit un parallèle avec la lutte contre le cancer et insiste sur le besoin d'un effort d'intelligence qui nous permettrait de dépasser les "dérives paresseuses, les nomenclatures désuètes, esprit de clocher, cloisonnement $»$. Il attire l'attention sur les risques que ferait courir la permanence des «processus d'érosion qui risquent - si nous n'y prenons pas garde - de le corrompre ». Cet effort d'intelligence prescrit par Yves Stourdzé, est aujourd'hui d'une actualité brulante avec l'importance de l'amélioration de l'information et de la communication et donc le déploiement d'infrastructures adaptées et appropriées. Yves Stourdzé a de plus identifié de façon lumineuse l'importance de l'échange avec les patients, vingt ans avant l'introduction des premiers textes réglementaires qui instaureront la démocratie sanitaire dans notre pays. «Intelligence au sein du rassemblement de l'information, intelligence de la surveillance, intelligence en terme d'analyse d'image, intelligence sous forme d'échange d'information, entre ceux qui travaillent à la mise au point des molécules, des machines, et ceux qui les mettent en cuvre. Intelligence enfin dans la relation entre l'homme malade soumis à la menace et ceux qui disposent des instruments de dissuasion et de destruction de son mal ». 
$\mathrm{N} \cdot \mathrm{O} \cdot \mathrm{T} \cdot \mathrm{E} \cdot \mathrm{S}$

1. René Rettig.

2. Jeanne Auzenet.

3. Carole Wieland.

4. Le $\mathrm{D}^{\mathrm{r}}$ Pierre Trotot, chef de service de radiologie.

5. Voir l'article du Monde de 2013 «Alerte sur la désinfection des sondes d'échographies », Juin 2013. http://www.lemonde.fr/sante/article/2013/06/03/ alerte-sur-la-desinfection-des-sondes-dechographie_3423042_1651302.html

6. Ils sont en passe d'être rejoints par le coréen Samsung qui a décidé d'investir ce secteur.

7. Alors que pour les Anglo-saxons le passage des barrières réglementaires spécifiques au monde de la santé était considéré comme un avantage compétitif et un moyen de valoriser les entreprises, la pensée dominante en France était de les considérer comme des handicaps. Trente ans plus tard, le constat d'une capture de la régulation par l'industrie, notamment nord-américaine est actuellement débattu. Le marché médical fait vivre l'industrie sans doute la plus puissante économiquement dans le monde contemporain.

8. Le $\mathrm{D}^{\mathrm{r}}$ Olivier Rabin qui intervient toujours sur ce sujet comme enseignant dans le cadre du Diplôme Universitaire de Valorisation de la recherche imaginé et lancé en 1982 par le Pr Alain Sézeur avec le concours de l'auteur. Le DU de valorisation de la recherche a accueilli à ce jour plus de 3000 professionnels intéressés par la valorisation. http://dugbm.ticemed. upmc.fr/

9. Jean-Marc Jancovici et Alain Feingold. Auteurs de la thèse sous la direction de Jacques Attali dans le cadre de l'option économie de Jacques Stoléru à l'école Polytechnique.

10. Notamment dans le cadre d'un rapport du Conseil
Economique Social et Environnemental, voir http:// www.lecese.fr/content/le-cese-se-prononcera-surla-place-des-dispositifs-medicaux-dans-la-strategienationale-de-sante

11. Par des penseurs proches de Donald Reagan mais il faudra attendre l'arrivée de Newt Gingrich au congrès pour que le gouvernement décide de supprimer définitivement l'OTA en 1995.

12. Aux États-Unis, elle est désormais placée au troisième rang derrière les maladies cardiovasculaires et le cancer. Les erreurs médicales évitables y seraient responsables de 400000 morts par an. Appliquée à la France, on aboutirait à estimer leur nombre à 60000 par an (soit plus de 20 fois la mortalité routière actuelle). 13. Pour une présentation du contexte et des enjeux de la médecine de précision, J.-P. Thierry, Les nouveaux paradigmes de la médecine personnalisée ou de précision. Enjeux juridiques, médicaux et éthiques, sous la direction de Christian Hervé et Michèle Stanton-Jean. Dalloz. Collection : Thèmes \& Commentaires, Novembre 2014. 


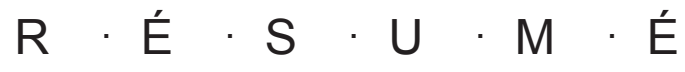

Le CESTA a permis de démarrer une activité de veille technologique et de prospective dans le domaine des technologies médicales à l'interface de compétences scientifiques et d'acteurs d'origines diverses [une petite équipe, composée d'une sociologue, d'un directeur d'hôpital et d'un médecin de santé publique et des interlocuteurs des ministères (santé et recherche notamment), d'experts médicaux issus de l'université et d'organismes de recherche et des industriels du secteur : groupes et PME]. L'organisation des séminaires «Technologies Nouvelles à l'Hôpital » était l'une des activités les plus visibles car elle débouchait sur l'organisation de réunions publiques qui permettaient de confronter les points de vue et surtout d'établir des synthèses susceptibles de clarifier les enjeux et les perspectives de diffusion des technologies nouvelles en médecine (imagerie médicale, ultrasons et échographie, biologie médicale, informatique). Ces réunions TNH ont permis au CESTA d'inscrire son action dans le cadre du « Technology Assessment » qui aurait pu, avec le temps, bénéficier d'une approche transdisciplinaire, notamment avec les biotechnologies, mais aussi pour la robotique, l'enseignement, les télécommunications. Rétrospectivement, on ne peut que s'interroger sur ce que la poursuite de cette activité assez unique aurait pu apporter au secteur des technologies médicales que la France, malgré quelques pépites et de rares fleurons, n'a pas su bien défendre. L'année de la fermeture du CESTA est aussi l'année où la Thomson CSF a décidé d'échanger sa branche médicale - la compagnie générale de radiologie contre l'activité de téléviseur de RCA. Il s'agissait du dernier groupe d'électronique français à se retirer de l'un des secteurs d'activité qui, sur longue période, s'est avéré comme l'un des plus rentables. Trente ans plus tard, dans un contexte qui rend plus que jamais nécessaire d'aider les acteurs en leur fournissant les éléments d'analyse (veille, prévision, prospective), un CESTA trouverait toujours toute sa place.

\section{Abstract}

CESTA was able to track and predict developments in medical technology at the interface of scientific expertise and the field's many different participants. It included among its members, a sociologist, a hospital director, a public health doctor, and experts from several ministries (most notably those of health and research), from universities, from research organizations and industry (corporations and SMEs). Organizing a seminar series entitled "New Technologies in the Hospital" was one of its highest profile activities, because this led to the holding of public meetings in which opposing points of view could be aired and what was at stake in the new medical technologies (medical imaging, ultrasounds and sonograms, medical biology, and computerized medicine) and their spread could be discussed together and clarified. The seminar gatherings enabled CESTA to include its activities under the rubric of "Technology Assessment," something that would have, over time, benefited from a transdisciplinary approach, not only in biotechnology but also in robotics, teaching, and telecommunications. In retrospect, we can only wonder what the pursuit of this unusual program might have brought to the medical technology sector, had France better fostered it. Despite producing some nuggets and even a few real gems, CESTA was closed down in the very year that Thomson CSF decided to give up its medical branch - its radiology company - in favor of working with RCA television. This was the last French electronics firm to withdraw from a sector which turned out, over time, to be exceptionally profitable. Thirty years later, in a context in which assisting players 
by providing them with analysis in such domains as monitoring, statistical modeling and forecasting is more needed than ever, an outfit like CESTA would still prove its worth. 